\title{
O aumento das doenças psicossomáticas durante a pandemia e dificuldades no
}

\section{atendimento psicológico}

\author{
The increase of psychosomatic diseases during the pandemic and difficulties in psychological care \\ El aumento de las enfermedades psicosomáticas durante la pandemia y las dificultades en la \\ atención psicológica
}

Recebido: 09/06/2021 | Revisado: 17/06/2021 | Aceito: 24/06/2021 | Publicado: 07/07/2021

\author{
Alana Gabriela de Araujo Passos \\ ORCID: https://orcid.org/0000-0002-1350-422X \\ Centro de Ensino Unificado do Piauí, Brasil \\ E-mail: alanagabriela1005.ag.ag@gmail.com \\ Glenda Silva Neto \\ ORCID: https://orcid.org/0000-0002-8276-667X \\ Centro de Ensino Unificado do Piauí, Brasil \\ E-mail: Glendag488@gmail.com \\ Iaira Milhomem de Araújo \\ ORCID: https://orcid.org/0000-0001-7237-8254 \\ Centro de Ensino Unificado do Piauí, Brasil \\ E-mail: iairamilhomem16@gmail.com \\ Maria Renata Alves Cardoso \\ ORCID: https://orcid.org/0000-0001-7968-7797 \\ Centro de Ensino Unificado do Piauí, Brasil \\ E-mail: mariarenata1245@outlook.com \\ Maria Samira Costa Alves \\ ORCID: https://orcid.org/0000-0002-2550-3179 \\ Centro de Ensino Unificado do Piauí, Brasil \\ E-mail: mariasamira2017@hotmail.com \\ Rita de Cássia de Almeida Silva \\ ORCID: https://orcid.org/0000-0002-4775-8017 \\ Centro de Ensino Unificado do Piauí, Brasil \\ E-mail: Ritadecassiaalm1@ Outlook.com \\ Tainara Sousa Pereira \\ ORCID: https://orcid.org/0000-0002-3158-3814 \\ Centro de Ensino Unificado do Piauí, Brasil \\ E-mail: thaynarasousa1371@gmail.com \\ Bruno da Silva Gomes \\ ORCID: https://orcid.org/0000-0002-4527-3956 \\ Centro de Ensino Unificado do Piauí, Brasil \\ E-mail: bsgomes100@gmail.com
}

\begin{abstract}
Resumo
Objetivo: explicar o crescente número de pessoas acometidas com transtornos psicossomáticos em período pandêmico, bem como a dificuldade em obter atendimento psicológico. Metodologia: trata-se de uma revisão bibliográfica, a partir dos artigos escolhidos teve seus resultados analisados e discutidos, apresentada a revisão do conteúdo abordado. De acordo com os seguintes critérios de inclusão: no idioma português, tendo como assunto principal doenças psicossomáticas; 07 artigos foram selecionados da base de dados do Google Acadêmico e SciELO. Destas 07 bibliografias estavam relacionados à temática e seguiram para a leitura minuciosa e posterior análise. Resultados: diante dos dados coletados, percebeu-se que durante a pandemia do novo coronavírus houve um aumento nas doenças psicossomáticas e nos níveis de ansiedade, depressão, dificuldades no sono e estresse, tendo como principais grupos afetados as mulheres e os profissionais da área da saúde, além disso, dificuldades no atendimento psicológico mostraram-se presentes em todos os setores. Conclusão: o estudo analisou que a pandemia trouxe muitos danos a saúde mental e física dos indivíduos, tendo como fatores a disseminação de notícias negativas ou falsas, além das perdas de entes queridos, e as dificuldades em atendimento psicológico, como a redução de serviços prestados no CAPS, o que gerou mais sofrimento mental em quase metade da população.
\end{abstract}

Palavras-chave: Doenças psicossomáticas; Pandemia; Isolamento social; Covid-19.

\section{Abstract}

Objective: to explain the growing number of people suffering from psychosomatic disorders in a pandemic period, as well as the difficulty in obtaining psychological care. Methodology: this is a literature review from the chosen articles, the results of which were analyzed and discussed, and a review of the content addressed was presented. 
According to the following inclusion criteria: in Portuguese, with psychosomatic diseases as the main subject; 07 articles were selected from the Google Scholar and SciELO database. Of these 07 bibliographies were related to the theme and followed for a thorough reading and further analysis. Results: in view of the data collected, it was noticed that during the pandemic of the new corona virus there was an increase in psychosomatic illnesses and in levels of anxiety, depression, sleep difficulties and stress, with women and professionals in the area being the main groups affected. health, in addition, difficulties in psychological care were present in all sectors. Conclusion: the study analyzed that the pandemic brought a lot of damage to the mental and physical health of individuals, having as factors the dissemination of negative or false news, in addition to the loss of loved ones, and difficulties in psychological care, such as the reduction of services provided in CAPS, which generated more mental suffering in almost half of the population.

Keywords: Psychosomatic diseases; Pandemic; Social isolation; Covid-19.

\section{Resumen}

Objetivo: explicar el creciente número de personas que padecen trastornos psicosomáticos en un período pandémico, así como la dificultad para obtener atención psicológica. Metodología: se trata de una revisión de la literatura a partir de los artículos seleccionados, cuyos resultados fueron analizados y discutidos, y se presentó una revisión de los contenidos abordados. Según los siguientes criterios de inclusión: en portugués, con enfermedades psicosomáticas como tema principal; Se seleccionaron 07 artículos de la base de datos Google Scholar y SciELO. De estas 07 bibliografías se relacionaron con el tema y se siguieron para una lectura exhaustiva y un análisis más profundo. Resultados: a la vista de los datos recolectados, se notó que durante la pandemia del nuevo coronavirus se registró un aumento de las enfermedades psicosomáticas y de los niveles de ansiedad, depresión, dificultades para dormir y estrés, siendo las mujeres y los profesionales del área los principales grupos afectados salud, además, las dificultades en la atención psicológica se presentaron en todos los sectores. Conclusión: el estudio analizó que la pandemia trajo mucho daño a la salud mental y física de las personas, teniendo como factores la difusión de noticias negativas o falsas, además de la pérdida de seres queridos, y dificultades en la atención psicológica, como la reducción de los servicios prestados en CAPS, que generó más sufrimiento mental en casi la mitad de la población.

Palabras clave: Enfermedades psicosomáticas; Pandemia; Aislamiento social; Covid-19.

\section{Introdução}

A Covid-19 é uma infecção respiratória ocasionada pelo SARS-Cov-2, de alto risco de propagação e tem afetado o mundo inteiro, a infecção tem produzidos quadros diferentes e alguns são considerados de riscos e outros de menor gravidade, em casos mais graves diversos sintomas são apresentados, desde a falta de ar até o comprometimento de partes do sistema respiratório, nos casos mais leves algumas pessoas tendem a não apresentar nenhum sintoma (Ministério da Saúde, 2021).

Devido à proliferação ser bastante contagiosa, o isolamento social foi necessário, provocando impactos na saúde mental dos indivíduos e um consequente aumento das doenças psicossomáticas. As doenças psicossomáticas são irregularidades emocionais ou psiquiátricas que produzem alterações no comportamento, e em diversos órgãos do corpo, sobre a relação mente-corpo, e a engrenagem de produção de enfermidades relacionada a fenômenos de estresse (Mello, 2010).

Nesse contexto, as mudanças de cenário alteram a atuação dos psicólogos e de psicólogas de todo o Brasil, trazendo dificuldades no atendimento psicológico, frente à crise o Conselho Federal de Psicologia (CFP) regulamenta a prestação de serviços psicológicos por meio de recursos tecnológicos e de comunicação em tempos de pandemia. Através da Resolução ${ }^{\circ}$ 11, de 11 de maio de 2018 que autoriza a prestação dos serviços psicológicos de maneira online (Conselho Federal de Psicologia, 2020).

O presente artigo objetiva explicar o crescente número de pessoas acometidas com transtornos psicossomáticos em período pandêmico, bem como a dificuldade em obter atendimento psicológico, por meio de uma revisão bibliográfica tem como foco entender o impacto do isolamento na saúde mental da população e consequências geradas por ele, enfatizando o quão maléfico fisicamente e mentalmente pode ser passar por esse período sem um acompanhamento psíquico adequado.

\section{Metodologia}

O início do estudo foi executado com a formação teórica sobre o tema, iniciando-se por uma pesquisa bibliográfica e análise preliminar sobre o tema de pesquisa, esse tipo de pesquisa consiste em analisar as fontes de dados da literatura sobre o tema (Sampaio \& Mancine, 2006). Quanto à realização desse estudo, a identificação do tema baseou-se no Aumento de Doenças Psicossomáticas, fator concludente para a seguinte delimitação: O aumento das Doenças Psicossomáticas em período de pandemia e dificuldades em atendimento psicológico. Em seguida, houve a identificação dos artigos pré-selecionados e selecionados, seguindo os critérios de inclusão. O próximo passo a partir dos artigos escolhidos teve seus resultados analisados e discutidos, apresentada a revisão do conteúdo abordado. De acordo com os seguintes critérios de inclusão: no idioma português, tendo como assunto principal Doenças Psicossomáticas; 13 artigos foram selecionados. Destes, 07 bibliografias 
estavam relacionadas à temática e seguiram para leitura minuciosa, e posterior análise. Com o propósito de uma maior compreensão da metodologia escolhida, a seguir foram apresentadas de modo sistemático cada etapa da Revisão Sistemática da Literatura utilizada para a elaboração deste estudo.

Na Figura 1, primeiramente, temos a definição do tema de estudo juntamente com a delimitação, posteriormente, está representado as bases de dados que foram retirados os artigo, a partir disso foi aplicado os critérios de inclusão e consequentemente selecionado 13 artigos, como está descrito no diagrama abaixo, e por fim, 07 bibliografias foram selecionadas para a revisão.

Figura 1. Diagrama com as etapas para a construção do artigo

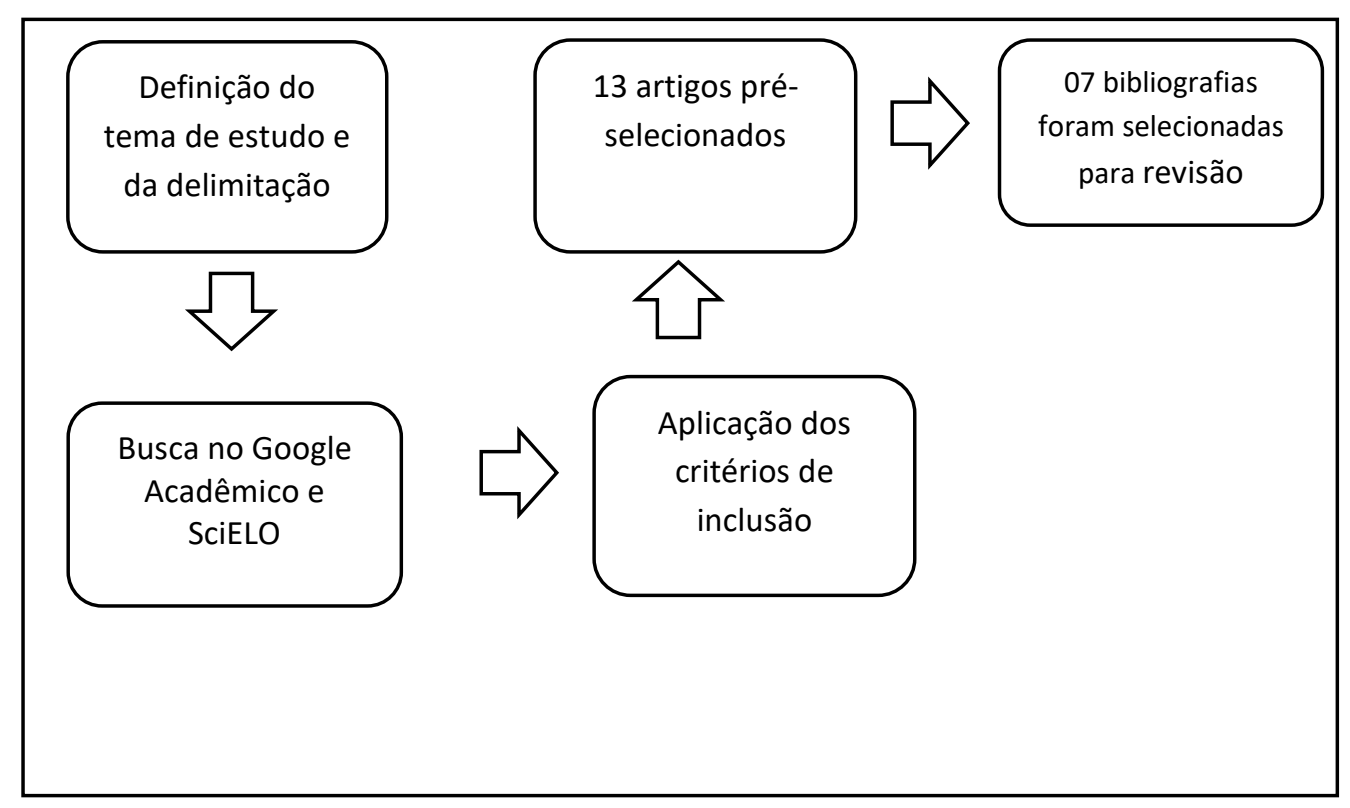

Fonte: Autores.

A Figura 1 representa todas as etapas necessárias para a produção do artigo, é importante observar que cada etapa está descrita de forma sucinta, deixando explícito o que foi feito em cada etapa.

\section{Resultados}

No Quadro 1, apresentam-se as características das publicações quanto ao ano, título, autores e principais considerações para o tema da pesquisa, sendo todos artigos científicos da base de dados do Google Acadêmico e SciELO. 
Quadro 1. Características das publicações quanto ao ano, título, autores e principais considerações.

\begin{tabular}{|c|c|c|c|c|}
\hline $\mathrm{N}^{\circ}$ & ANO & TÍTULO & AUTORES & PRINCIPAIS CONSIDERAÇÕES \\
\hline $\mathrm{A} 1$ & 2019 & $\begin{array}{l}\text { Manifestações corporais do } \\
\text { sofrimento psíquico: psicossomática } \\
\text { em contexto de pronto socorro. }\end{array}$ & MILHORIM; COSTA & $\begin{array}{l}\text { As perdas e o processo de luto podem favorecer para } \\
\text { surgimentos de sinais e sintomas psicossomáticos. }\end{array}$ \\
\hline $\mathrm{A} 2$ & 2020 & $\begin{array}{l}\text { Saúde mental em tempos de Covid- } \\
\text { 19: a importância do atendimento } \\
\text { psicológico remoto. }\end{array}$ & SANTOS; OLIVEIRA & $\begin{array}{l}\text { O estudo mostra a importância do atendimento online durante } \\
\text { a pandemia para lidar melhor com a ansiedade, estresse e } \\
\text { depressão, além do desenvolvimento do mesmo para um } \\
\text { período pós-pandêmico. }\end{array}$ \\
\hline A3 & 2020 & $\begin{array}{l}\text { Relato de tristeza/depressão, } \\
\text { nervosismo/ansiedade, e problemas } \\
\text { de sono na população adulta } \\
\text { brasileira durante a pandemia de } \\
\text { Covid-19 }\end{array}$ & BARROS et al. & $\begin{array}{l}\text { A pandemia aumentou os níveis de ansiedade, nervosismo, } \\
\text { depressão, tristeza e dificuldades no sono da população } \\
\text { brasileira. }\end{array}$ \\
\hline A4 & 2020 & $\begin{array}{l}\text { Atuação do psicólogo no Centro de } \\
\text { Atenção Psicossocial (CAPS) em } \\
\text { tempos de pandemia Covid-19. }\end{array}$ & BRUN; CANEDA & $\begin{array}{l}\text { A pandemia trouxe dificuldades na atuação do psicólogo no } \\
\text { CAPS. }\end{array}$ \\
\hline A5 & 2020 & $\begin{array}{l}\text { Covid-19 em profissionais da saúde, } \\
\text { vivências e perspectivas: um relato } \\
\text { de experiência. }\end{array}$ & FIGUEIREDO et al. & $\begin{array}{l}\text { Os profissionais no período da pandemia relataram reações } \\
\text { psicossomáticas semelhantes aos sinais e sintomas da Covid- } \\
19 .\end{array}$ \\
\hline A6 & 2020 & $\begin{array}{l}\text { Incidência de sintomas de ansiedade } \\
\text { em profissionais da área da saúde } \\
\text { atuantes no combate à pandemia da } \\
\text { Covid-19. }\end{array}$ & SOUZA; ALMEIDA & $\begin{array}{l}\text { Os sintomas mais graves detectados na pesquisa com } \\
\text { profissionais de saúde foram acelerações no coração e } \\
\text { desconforto no abdômen. }\end{array}$ \\
\hline A7 & 2021 & $\begin{array}{l}\text { Caracterização e demanda de um } \\
\text { serviço de atendimento psicológico } \\
\text { on-line no contexto da pandemia de } \\
\text { Covid-19. }\end{array}$ & $\begin{array}{l}\text { PINTO; SANTOS; } \\
\text { ZAMPIERI }\end{array}$ & $\begin{array}{l}\text { No período de abril a novembro de } 2020 \text { foram atendidas de } \\
\text { forma online } 145 \text { pessoas, sendo a maioria mulheres e adultos } \\
\text { intermediários, no qual se percebeu que as maiores queixas } \\
\text { eram ansiedade, depressão, dificuldade em lidar com o } \\
\text { isolamento e problemas familiares. }\end{array}$ \\
\hline
\end{tabular}

Fonte: Autores.

\section{Discussão}

A pandemia do Covid-19 afetou a qualidade de vida da população em geral (Figueiredo et al., 2020), uma pesquisa realizada com amostra de 45.161 brasileiros por meio de um formulário, identificou que quase metade da população sentia-se triste ou com sinais de depressão e metade afirmaram ter sintomas de ansiedade, as mulheres sentiam-se duas vezes mais ansiosas e depressivas do que os homens, a prevalência desses resultados são devido as mesmas serem mais atentas a saúde e mostrarem-se mais alertas a sinais e sintomas, além de sobrecarga por estarem cuidando de crianças e trabalhando em casa (Barros et al., 2020).

Além da população em geral, um grupo que apresentou a saúde mental abalada foram os profissionais da área da saúde, no qual houve presença de sinais e sintomas de ansiedade e de doenças psicossomáticas (Souza \& Almeida, 2020). Além disso, um sinal psicossomático observado foi à presença de sintomas semelhantes com os da Covid-19, sendo que os profissionais não estavam com a doença. As causas para tais aflições são o desconhecimento da pandemia, sendo algo nunca visto antes pelos profissionais, medo de transmitir a doença para seus familiares, já que mantem contato direto com os infectados e dificuldades no isolamento (Figueiredo et al., 2020).

Nesse contexto, as doenças psicossomáticas se mostraram presentes na população brasileira durante a pandemia do Covid-19, os resultados mostraram que sinais e sintomas sem uma causa específica foram identificados, tais como: aceleração no coração, desconforto no abdômen (Souza \& Almeida, 2020), reações semelhantes com os sintomas de Covid-19 (Figueiredo et al, 2020), além de sintomas de ansiedade, depressão, estresse e dificuldades no sono aumentaram durante a pandemia (Pinto, Santos, \& Zampieri, 2021). Um dos fatores que contribuem para a presença desses sinais e sintomas são as informações negativas que as pessoas são submetidas diariamente e a incerteza da confiabilidade das mesmas (Santos \& Oliveira, 2020). Ademais, a perda de entes queridos e a falta de contato com o falecido para uma vivência do luto contribui para um aumento de doenças psicossomáticas (Milhorim \& Costa, 2019), dessa forma para evitar uma maior propagação da doença, os indivíduos acabam não se despedindo dos entes queridos, produzindo consequências para os mesmos.

Diante do exposto, devido à complexidade da pandemia e o impacto trazido na saúde mental dos indivíduos e aumento das doenças psicossomáticas, dificuldades no atendimento psicológico foram encontradas. O despreparo dos sistemas públicos e privados para a pandemia é um dos problemas enfrentados (Figueiredo et al., 2020), o desconhecimento da doença e 
velocidade do contágio contribui para essa problemática. O aumento da procura por serviços psicológicos também se mostrou um impasse, mas devido o isolamento social, novos recursos se apresentaram eficientes para o contexto, como o atendimento online (Pinto, Santos, \& Zampieri, 2021), no entanto o atendimento deve seguir os parâmetros estabelecidos pelo o Conselho Federal de Psicologia (CFP), o que acaba trazendo algumas limitações em relação aos casos que podem ser atendidos na modalidade online (Santos \& Oliveira, 2020), em relação à demanda elevada, pode-se considerar ainda, a sobrecarga presente nos profissionais, falta de profissionais e a modificação no ambiente de trabalho, sendo necessário um processo de adaptação.

Além disso, vale salientar que o atendimento psicológico vai além de serviços em consultórios e envolve uma rede de atendimentos (Santos \& Oliveira, 2020), um desses serviços é o Centro de Atenção Psicossocial (CAPS) que com a pandemia acabou por ter prejuízos no seu funcionamento. Nesse período, houve a suspenção de programas, oficinas terapêuticas e grupos de encontro, havendo uma limitação nas atividades desenvolvidas, deixando apenas as essenciais (Brun \& Caneda, 2020), por meio disso observa-se uma problemática envolvida, pois a população que não pode pagar por um serviço particular de atendido faz uso do CAPS em busca de apoio e acolhimento e essas limitações acabam por trazer prejuízos para essas pessoas.

\section{Conclusão}

O presente estudo observou a presença de aspectos psicológicos e sua relação com a eclosão das doenças psicossomáticas, foi identificado que quase metade da população sentia-se triste e com sinais de depressão. Além da população em geral, outra área bastante afetada foram os profissionais da saúde, que estão em contato direto com pessoas infectadas e correm o risco de contrair o vírus e levar para casa, muitos indivíduos chegaram a adoecer pela disseminação de notícias negativas no qual são expostos diariamente, o que acaba gerando um grande estresse.

A Covid-19 trouxe muitos danos à saúde mental e física dos indivíduos, como o aumento das doenças psicossomáticas, tem a questão da dificuldade em atendimento psicológico, pois os CAPS tiveram que ter suas atividades reduzidas, muitos profissionais pararam os atendimentos o que elevou bastante o número de pessoas com sofrimento mental.

Saber ao certo quais implicações trouxeram essas dificuldades no atendimento psicológico, o impacto que o aumento das doenças psicossomáticas refletiu na sociedade, entender quais fatores da pandemia mais afetaram a população, pois ainda é algo muito amplo, envolve várias questões e não sabemos ao certo, sempre sofre influência de vários fatores, sempre uma fator puxa o outro, mas não sabemos qual deles desencadeia uma maior resposta.

\section{Referências}

Barros, M. B. A., Lima, M. G., Malta, D. C., Szwarcwald, C. L., Azevedo, R. C. S., Romero, D., Souza, P. R. B. Jr., Azevedo, L. O., Machado, I. E., Damacena, G. N., Gomes, C. S., Werneck, A. O., Silva, D. R. P., Pina, M. F., \& Gracie, R. (2020). Relato de tristeza/depressão, nervosismo/ansiedade e problemas de sono na população adulta brasileira durante a pandemia de COVID-19. Revista de Epidemiologia e Serviços de Saúde, 29 (e2020427), 1-12. $10.1590 /$ S1679-49742020000400018.

Conselho Federal de Psicologia. (2020). Resolução $N^{\circ} 4$, de 26 de março de 2020. http://crp11.org.br/upload/Resolu\%C3\%A7\%C3

$\%$ A3o\%20CFP\%204\%202020.

Ministério da Saúde. (2021). Coronavírus (COVID-19). https://coronavirus.saude.gov.br/sobre-a-doenca.

Brum, H. K., \& Gonçalves, C. R. (2020). Atuação do psicólogo no centro de atenção psicossocial (CAPS) em tempos de pandemia Covid-19. Anais do (Inter) Faces, 1 (1). https://www.ulbracds.com.br/index.php/interfaces/article/view/2905

Figueiredo, A. M., Jr., Calandrine, E. F., Sousa, Y. M., Galvão, M. M., Cunha, F. F., Veras, A. S. F, Gonçalves, W.O. F., Mesquita, D. S., Costa, J. V. T., \& Silva, C. K. A. (2020). COVID-19 em profissionais da saúde, vivências e perspectivas: um relato de experiência. Revista Eletrônica Acervo Saúde, 12 (12), 15. https://doi.org/10.25248/reas.e5258.2020

Mello, J. F. (2010). Psicossomática hoje. Artmed.

Milhorim, T. K., \& Costa, S. B. N. (2019). Manifestações corporais do sofrimento psíquico: psicossomática em contexto de pronto-socorro. Revista SBPH, 22 (1), 127-153. de http://pepsic.bvsalud.org/scielo.php?script=sci_arttext\&pid=S1516-08582019000100008

Pinto, A. A., Jr., Santos, M. S. M. P., \& Zampieri, T. C. R. C. (2021). Caracterização e demanda de um serviço de atendimento psicológico on-line no contexto da pandemia de Covid-19. Revista de Psicologia e Saúde em Debate, 7 (1), 94-106. https://doi.org/10.22289/2446-922X.V7N1 A7

Sampaio, R. P., Mancini, M. C. (2006). Estudos de Revisão Sistemática: um guia para a síntese criteriosa da evidência científica. Revista Brasileira de Fisioterapia, 11 (1), 83-89. https://doi.org/10.1590/S1413-35552007000100013

Santos, M. F. R., \& Oliveira, M. E. F. (2020). Saúde Mental em tempos de Covid-19: a importância do atendimento psicológico remoto. Revista Transformar, 14 (2), 76-90. http://www.fsj.edu.br/transformar/index.php/transformar/article/view/378

Souza, M. N., \& Almeida, M. P. P. M. (2020). Incidência de sintomas de ansiedade em profissionais da área da saúde atuantes no combate à pandemia da Covid-19. Psicologia-Tubarão. http://www.riuni.unisul.br/handle/12345/11665 